\title{
Assessment of Patient Counseling on Dispensing of Medicines in Outpatient Pharmacy of Tikur-Anbessa Specialized Hospital, Ethiopia
}

\author{
Sara Ali ${ }^{1}$, Tariku Shimels ${ }^{* 2}$, and Arebu I. Bilal ${ }^{1}$
}

OPEN ACCESS

Citation: Sara Ali, Tariku Shimels, and Arebu I. Bilal. Assessment of Patient Counseling on Dispensing of Medicines in Outpatient Pharmacy of Tikur-Anbessa Specialized Hospital, Ethiopia. Ethiop J Health Sci. 2019; 29(6):727.doi:http://dx.doi.org/10.4314/ej hs.v29 i6.9

Received: February 27, 2019

Accepted: July 23, 2019

Published: November 1, 2019

Copyright: (C2019 Sara A, et al. This is an open access article distributed under the terms of the Creative Commons Attribution License, which permits unrestricted use, distribution, and reproduction in any medium, provided the original author and source are credited. Funding: Nil

Competing Interests: The authors declare that this manuscript was approved by all authors in its form and that no competing interest exists.

Affiliation and Correspondence:

${ }^{1}$ Addis Ababa University, College of

Health Sciences, School of Pharmacy,

Department of Pharmaceutics and

Social Pharmacy

Email; Arebu Issa:

issaareb@gmail.com, Sara Ali: afutiali27@gmail.com

${ }^{2}$ St. Paul's Hospital Millennium Medical College Research Directorate,

Addis Ababa, Ethiopia

*Email: tarphar2008@gmail.com
ABSTRACT

Background: Patients' care when dispensing of Medicines could be ensured through the delivery of drug information either orally or in written form. Pharmacists are expected to ascertain that patients or caregivers have gained clear and complete information. The objective of this study was to assess patient counseling during dispensing of medicines and associated factors in Tikur-Anbessa Specialized Hospital.

Method: A cross sectional study was conducted to assess patients' counseling and a convenient sampling technique was employed to select study participants. Data was collected using a structured questionnaire and observation checklist and, analyzed by using SPSS version 20. Descriptive statistics and odds ratio were used to present study results.

Results: A total of 286 respondents were included in this study. Most (93.7\%; 94.4\%; 91\%) of the respondents were told the drug unit dose, frequency of administration and duration of therapy respectively. Less than a third of the patients received information on drug-drug interactions, storage of medications and side -effects. None of the patients were told what to do on missed dose and, none were asked back to repeat their understanding. Three fourth of the patients reported that they were satisfied with the counseling services provided. Females were more likely to be satisfied than males whereas, level of education showed a negative association with pharmacist counseling services.

Conclusion: Drug dose, frequency of administration and duration of therapy were the most frequently delivered information to patients. Gender and level of education have significant association with medication counseling service in the study setting.

Key words: Counseling, OPD Pharmacy, Tikur-Anbessa Specialized Hospital

\section{INTRODUCTION}

A Proper medication dispensing practice encompasses; the delivery of the correct medicine to the right patient, ensuring the required dosage and quantities, maintaining a package that preserves the potency and quality of the drugs for the specified period, clear medicine information counseling and appropriate follow up (1). 
Having this general rule in mind, rational drug use also maintains the fact that patients do have individual requirements in terms of the earlier considerations to be aligned with their clinical needs and, at the lowest cost to them as well as to their community (2).

Apart from other Outpatient Department (OPD) services, Pharmacy departments have also key roles in delivering medication counseling at exit points. Among the main activities in such services include; a pharmacist-oriented prescription screening, drug filling for follow patients, dispensing as per standards, and stock management of medications (3). An appropriate drug counseling and dispensing is a component of pharmacy practice and, its need and impact on patientcare has gained particular importance as Pharmacists are usually the final link between the medication and the patient (4).

Expanding of the medication dispensing practice to integrate counseling and education to patients at both institutions and community pharmacies has been shown to be the main part of pharmacists' responsibility as pharmaceutical care providers (5). The need for proper counseling at pharmacies emanates from the fact that most drugrelated services are provided by pharmacy professionals (6) which demands their involvement to provide appropriate, understandable and relevant information to patients about their medication (7).

A good patient-provider relationship is an important indicator for desired medication outcomes and a basis for rational use of drugs (4, 5). The pharmacy professional should, hence, educate patients on the name and description of their medication, the purpose why the drug is prescribed for the patient, its dose, frequency of use, duration of therapy, directions and precautions for use of drugs, common side effects, contra-indications, proper storage, and appropriate actions to be considered on missed doses. Likely, possible drug-drug as well as drug-food interactions that could possibly occur should also be told verbally and accompanied by written information to patients $(2,8)$.

The information provided is meant to increase knowledge of patients about their medication and create awareness on proper adherence. Therefore, pharmacy professionals must take in to account that the information is given in a language that patients understand, and be checked whether or not patients have understood it clearly by letting them to repeat it in their own word (1). This helps understand patients' concerns and guide to deliver patientcentered care responsively and respectfully $(1,2$, $9,10)$. Despite a pharmacist mediated counseling is reported to prevent the occurrence of potential ADEs (11, 12), drug therapy problems and recommend cost-effective alternatives (13), it is often little practiced in the general pharmacy services $(14,15)$ leading to poor patientcare and service satisfaction. Among the frequently reported reasons of inappropriate medication counseling include; low level of medication knowledge (16), lack of counseling skills (14), work experience and work load (17) among others. In order to improve patient health care and reduce the number of medication related errors, a greater emphasis must be placed on pharmacists' patient counseling. Especially, in situations where pharmacy work force may not address adequate patient satisfaction, a remarkable emphasis must be given to retain better patientcare practice (18).

Even though certain medication counseling assessments have been conducted in different parts of Ethiopia $(6,15,17,19,20)$, there, still, exists variation in terms of the methodologies employed, the study populations considered and, contexts in which these studies were done. Accordingly, an assessment that combines both observation checklists and exit interview of patients was sought to address both pharmacy practices and knowledge of participants in medication counseling. The objective of this study was, therefore, to assess patient medication counseling practices during dispensing of medicines by pharmacy professionals in the outpatient pharmacy of Tikur-Anbessa Specialized Hospital (TASH), one of the tertiary care teaching hospitals in Ethiopia.

\section{MATERIALS AND METHODS}

Study area and period: This study was conducted in the outpatient pharmacy of TASH found in Addis Ababa, Ethiopia. The hospital has more than 600 beds and more than 250,000 outpatients visiting in a

DOI: http://dx.doi.org/10.4314/ejhs.v29i6.9 
year. The study was conducted from February to June 2018.

Study design: A Cross-sectional study was conducted by using an interviewer administered questionnaire and medication counseling observation checklist. Contents of the questionnaire were sociodemographic details of the patients, medication counseling information and patient's satisfaction as well as problems on counseling services. Main patient medication counseling information considered in this study included; dose and frequency of a drugs, timing of its use or administration, duration to taking the medicines, how to take medicines, how to store medicines, information on not to share drugs with other persons, type of foods or beverages to be avoided, demonstration of administration or application of certain drugs and, information on what to do when missing doses as well as when intended effect is not obtained among others.

Source and study population: The source population was all the patients of TASH who visited or have been attending to the different departments of the hospital whereas, the study population was all the outpatients who visited respective departments and finally attended the OPD pharmacy counters during the study period. The later was the population from whom the samples were drawn. Most of the outpatients visiting the OPD pharmacy of TASH refill medications for treating different chronic illnesses.

Sample Size: Single population proportion formula was used to calculate the sample size. After adjusting using a finite population correction factor, the final sample size was 338 patients and/or caregivers. As a patient was to be enrolled both for medication counseling observation and service satisfaction exit interview, convenient sampling technique was used to select the study units.

Data Collection Process and Method: Data was collected in two steps; first by observing pharmacy professional medication counseling practices at dispensary counters using a checklist and, second by subsequent exit interviewing of patients on counseling service satisfaction, perceived problems during dispensing and knowledge on the medications dispensed. Close ended questions were presented to participants in the second phase as part of the exit interview. A patient was included in the second exit interview if provided verbal consent in the earlier part of the data collection and was willing to participate in the interview. A structured patient care indicator and medication counseling checklist, adapted from WHO (21), was used to collect the information on patient medication counseling. Mean dispensing time was computed based on the individual patient's time to receive medication counseling at the pharmacy counters exempting earlier and later waiting times. Medication labeling and exit patient knowledge on medications was assumed adequate if it consists at least the dose, frequency and how to administer (take) the medicine.

Data Analysis: The collected data was cleared, categorized, and coded. All data collected were then analyzed using the Statistical Package for the Social Sciences (SPSS), version 20.0 software. Descriptive statistics was used to analyze participant characteristics, satisfaction with counseling services problems related to counseling. A binary logistics regression was used to determine factors associated with counseling service satisfaction. A P-value of 0.2 in the bivariate analysis was considered to be statistically significant for inclusion in the multivariable analysis. In the second model, statistical significance was considered at P-value of 0.05 and $95 \%$ confidence interval.

Ethical clearance for doing this study was obtained from AAU, school of pharmacy. The purpose of the study was clearly explained for the study participants. Information was collected after obtaining verbal consent from each participant. Study participants were told that they have the right to refuse or discontinue participation at any time they want and the chance to ask any thing about the study. For the purpose of maintaining confidentiality, a participant's name was not used at the time of data collection. In addition, all other personal information was kept entirely anonymous throughout the study period.

\section{RESULTS}

A total of 286 respondents were successfully included in both the medication counseling observation and the subsequent interview which gives an $85 \%$ response rate. From this, $176(61.5 \%)$ were males and $110(38.5 \%)$ were females. Most of the respondents included in this study were in the age group of $31-40(28 \%)$ and $41-50(21.7 \%)$. Two third of the study participants were married and most (70.6\%) were orthodox Christian followers (table 1).

DOI: http://dx.doi.org/10.4314/ejhs.v29i6.9 
Table 1: Socio demographic characteristics of the patients at TASH Outpatient Pharmacy, February 2018.

\begin{tabular}{|c|c|}
\hline Variable & $\mathbf{N}(\%)$ \\
\hline \multicolumn{2}{|l|}{ Gender } \\
\hline Male & $176(61.5)$ \\
\hline Female & $110(38.5)$ \\
\hline \multicolumn{2}{|l|}{ Age } \\
\hline 30 or less & $78(27.2)$ \\
\hline $31-50$ & $142(49.7)$ \\
\hline 51 or more & $66(23.1)$ \\
\hline \multicolumn{2}{|l|}{ Regions } \\
\hline A.A & $187(65.4)$ \\
\hline Amhara & $16(5.6)$ \\
\hline Somalia & $2(0.7)$ \\
\hline Oromia & $63(22.0)$ \\
\hline SNNPR & $18(6.3)$ \\
\hline \multicolumn{2}{|l|}{ Religion } \\
\hline Muslim & $56(19.6)$ \\
\hline Orthodox Christian & $202(70.6)$ \\
\hline Protestant Christian & $22(7.7)$ \\
\hline Others & $3(1.1)$ \\
\hline \multicolumn{2}{|l|}{ Educational status } \\
\hline Can or Can't read and write & $32(11.2)$ \\
\hline Grade $1-8$ & $86(30.1)$ \\
\hline Grade 9-12 & $104(36.3)$ \\
\hline University or College & $64(22.4)$ \\
\hline \multicolumn{2}{|l|}{ Marital status } \\
\hline Married & $194(67.8 \%)$ \\
\hline Single & $74(25.9 \%)$ \\
\hline Divorced & $6(2.1 \%)$ \\
\hline Widowed & $12(4.2 \%)$ \\
\hline \multicolumn{2}{|l|}{ Household size } \\
\hline Less than 6 & $220(76.9 \%)$ \\
\hline $6-10$ & $64(22.4 \%)$ \\
\hline Greater than 10 & $2(0.7 \%)$ \\
\hline \multicolumn{2}{|l|}{ Do you have a job? } \\
\hline Yes & $142(49.7 \%)$ \\
\hline No & $144(50.3 \%)$ \\
\hline \multicolumn{2}{|l|}{ Working sector } \\
\hline Government & $89(62.7 \%)$ \\
\hline Private & $53(37.3 \%)$ \\
\hline \multicolumn{2}{|l|}{ Average Monthly Income } \\
\hline 1000 or less & $18(12.7 \%)$ \\
\hline $1001-5000$ & $98(69.0 \%)$ \\
\hline More than 5000 & $26(18.3 \%)$ \\
\hline \multicolumn{2}{|l|}{ Work Experience } \\
\hline 10 or less & $54(38.0 \%)$ \\
\hline $11-20$ & $38(26.8 \%)$ \\
\hline $21-30$ & $32(22.5 \%)$ \\
\hline $31-40$ & $8(5.6 \%)$ \\
\hline 41 or more & $10(7.0 \%)$ \\
\hline
\end{tabular}

Most $(93.7 \% ; 94.4 \% ; 91 \%)$ of the respondents were told about the drug unit dose, frequency of administration and duration of therapy respectively. Less than a third proportion of the patients received information on drug-drug interaction, storage of medications, and major side effects. None of the patients were told what to do on missed dose and, none were asked back to repeat their understanding (Fig 1).

Patients' satisfaction with the counseling services: Three fourth of the patients reported that they were satisfied with the counseling services provided by TASH outpatients pharmacy. However, one fourth of the patients were unsatisfied with the counseling services provided by TASH (Fig. 2).

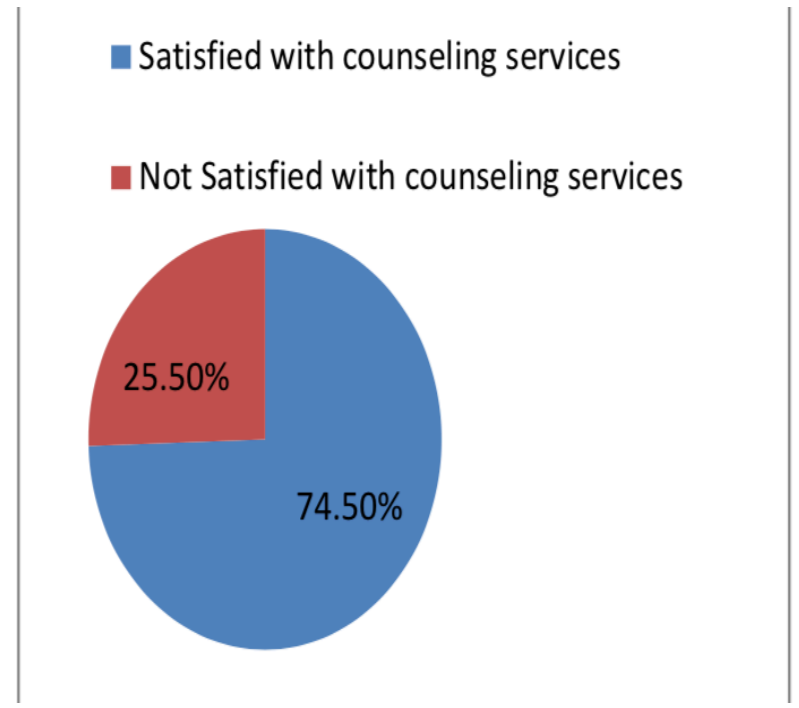

Figure 2: Percentage of patients satisfied with medication counseling service of TASH outpatient pharmacy, February 2018.

A total number of 852 drugs were prescribed on 286 prescriptions which give the number of drugs per prescription to be three. Of the 852 drugs prescribed, 674(79.1\%) were actually dispensed to patients (Fig. 3). 


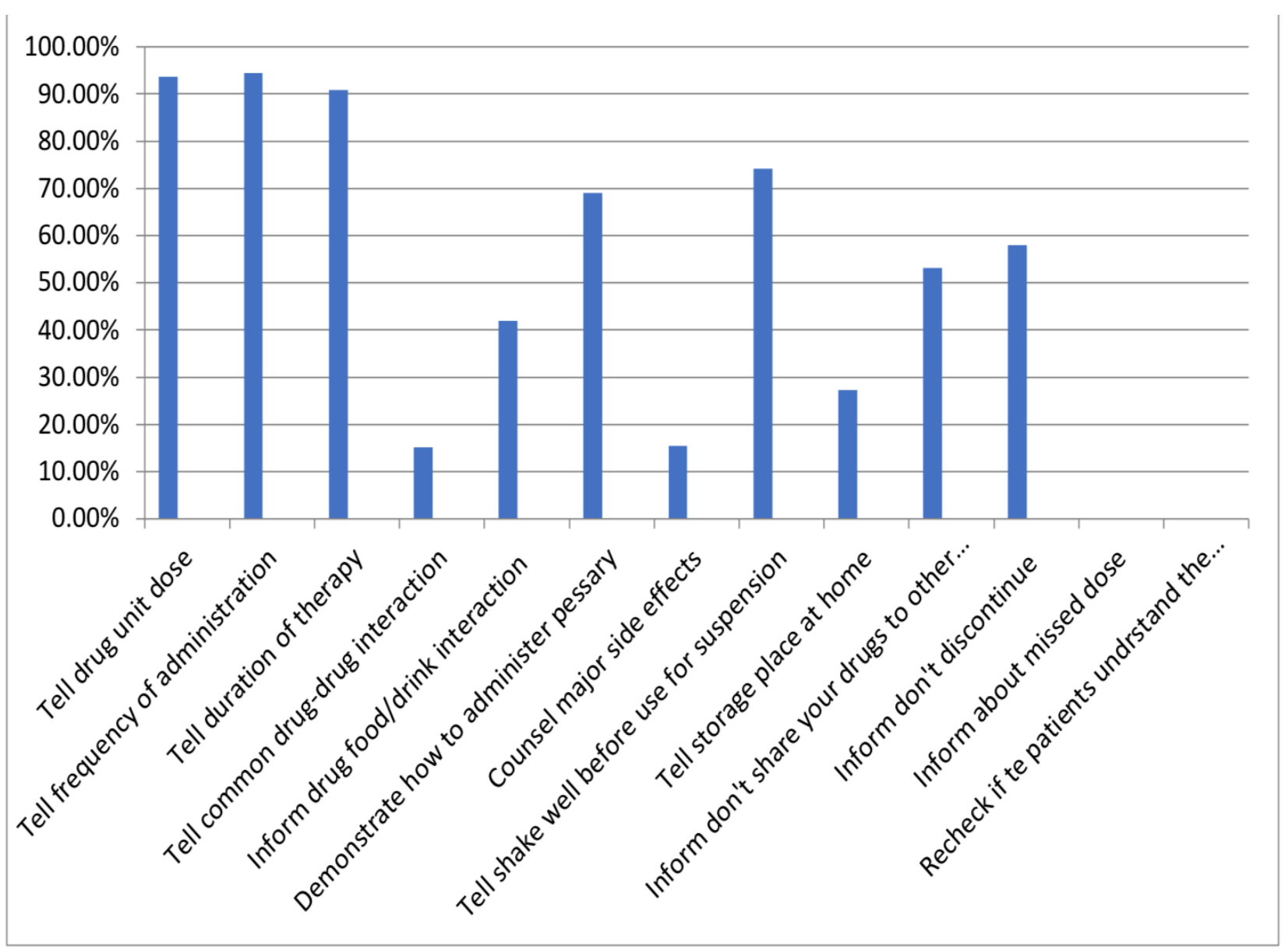

Figure 1: Percentage of patients who get the different counseling services at TASH outpatient pharmacy, February 2018.

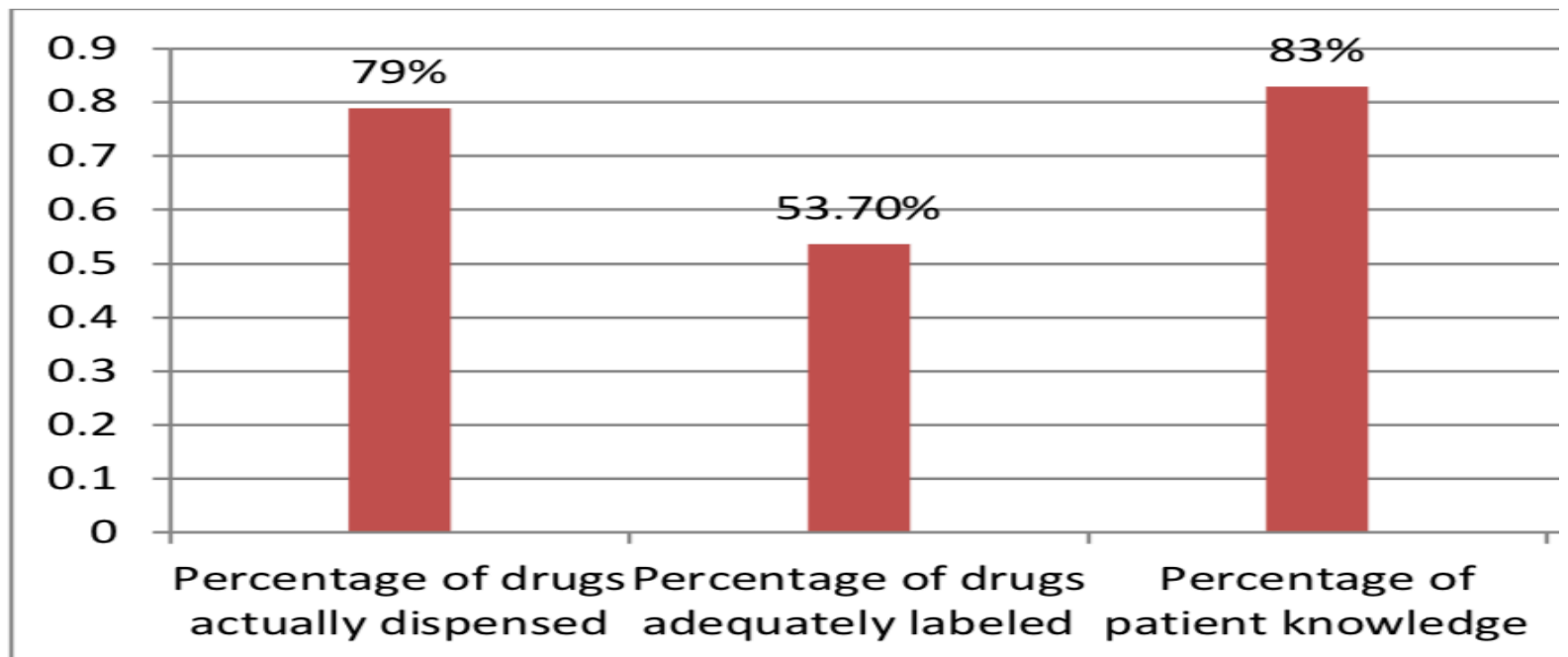

Figure 1: Percentage of patient care indicators at TASH, February 2018. 
Mean dispensing time, which is the most important component of the dispensing indicators, was conventionally used as a core dispensing indicator. The mean dispensing time spent to patients was found to be 53 seconds which is too short for the dispenser to pass the essential information to the patients or patients' care givers.

Problems related to counseling: Patients were asked whether they encountered problems during their visit of the outpatient pharmacy of TASH. Majority $(70.6 \%)$ of the respondents believe that

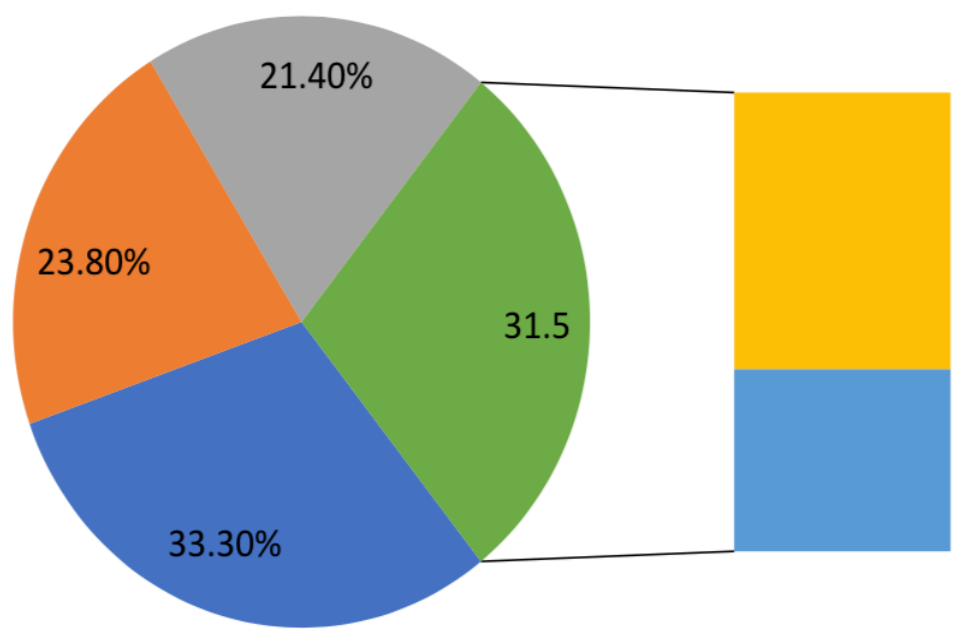

there is no problem of counseling. However, around $30 \%$ of the respondents agreed that there is a problem of counseling and have indicated some of the problems related to the counseling services in TASH (Figure 4). About a third of the patients $(33.3 \%)$ mentioned patient related factors (either lack of patience to listen the counseling provided or poor attitude to pharmacy services) as a main problem followed by those who mentioned patient load and lack of space.

Absence of legal frame-work to monitor and evaluate patient counseling practice

- High patient load

$19.5 \%$

Lack of space

Lack of updated drug information

$12.0 \%$

Patient factors

Figure 4: Problems related to medication counseling in TASH outpatient pharmacy, February 2018.

Factors associated with overall satisfaction of counseling services: Selected independent patient characteristics namely; gender, age, religion, education, work sector, marital status, income level and patient load were tested by a binary logistics regression for potential association with overall satisfaction of pharmacist mediated counseling services. Gender of respondents, age and level of education were found to have statistical significance and, were subsequently entered for the multivariable logistics regression analysis. Female gender was found to show a twofold likelihood of being satisfied by the counseling services. However, as the level of education level increases, respondents were less likely to have satisfaction from the counseling services in TASH pharmacy (Table 2). 
Table 2: Factors associated with overall satisfaction of pharmacist counseling services in TASH outpatient Pharmacy, February 2018.

\begin{tabular}{llllll}
\hline Variable & & \multicolumn{2}{c}{ Overall Satisfaction } & \multicolumn{2}{c}{ Odds Ratio } \\
\cline { 3 - 6 } & & & & Crude & Adjusted \\
& & Yes (N/\%) & No (N/\%) & $(95 \% \mathrm{CI})$ & $(95 \% \mathrm{CI})$ \\
\hline Gender & Female & $144(67.3)$ & $32(44.4)$ & $2.57(1.50-4.44)$ & $2.33(1.31-4.13)$ \\
& Male & $70(32.7)$ & $40(55.6)$ & 1.00 & 1.00 \\
Age (yrs.) & $<=30$ & $52(24.3)$ & $26(36.1)$ & $0.54(0.25-1.15)$ & $0.56(0.26-1.23)$ \\
& $31-50$ & $110(51.4)$ & $32(44.4)$ & $0.93(0.45-1.88)$ & $0.82(0.39-1.74)$ \\
Level of & $>50$ & $52(24.3)$ & $14(19.4)$ & 1.00 & 1.00 \\
Education & Can or cannot & $30((14.0)$ & $2(2.8)$ & 1.00 & 1.00 \\
& read \& write & & & & \\
& Grade 1-8 & $66(30.8)$ & $20(27.8)$ & $0.22(0.05-1.00)$ & $0.26(0.06-1.21)$ \\
& Grade 9-12 & $76(35.5)$ & $28(38.9)$ & $0.18(0.04-0.81)$ & $0.20(0.04-0.92)$ \\
& College or & $42(19.6)$ & $22(30.6)$ & $0.13(0.03-0.58)$ & $0.16(0.03-0.75)$ \\
& University & & & & \\
\hline
\end{tabular}

\section{DISCUSSION}

Counseling on medications is the best means among a variety of approaches that aid patients to achieve appropriate adherence on their drug therapy. Non- compliance to drug therapy is a common issue across the globe that potentially ends up with adverse drug reaction and therapeutic failure. It is part of the standard healthcare service and acknowledged by concerned authorities that medication counseling mediated by pharmacy professionals can significantly improve patients' awareness about medications and life style modification. In other words, failure of meeting treatment goals and reduction in quality of life of patients often follows poor adherence which, in turn, is linked to lack of adequate information on appropriate drug utilization (22).

In the present study, the drug unit dose, frequency of administration \& duration of therapy were the most commonly told information to patients. Similar findings were also reported from a study conducted in Jimma (23). A study done in Saudi Arabia has documented that medication dose was the most common type of information provided by pharmacists (24). An observational study done in Pakistan has mentioned that dose and frequency of the medicines were more commonly communicated to patients than any other information during drug dispensing (25).

Even though there were many aspects of drug counseling information to be delivered to patients along with medications, the practice in the present study has shown that most of the patients were not told far beyond telling the dose and frequency of administration. Common drug-drug interaction, major side effects \& storage place at home were informed in about a third of the patients, while none of the patients were told what to do on missed dose as well as asked back to repeat their understanding.

In this study, three fourth of the patients have reported that they were satisfied with the counseling services provided by TASH outpatient pharmacy services. The percentage of satisfaction in this study is greater than a finding in Bahir Dar where the rate of satisfaction was about 33\% (10). This difference could, possibly, be attributed to contextual variation in terms of the study setting and populations considered in the two studies. However, the figure in the present study is lower as compared to a finding obtained in Botswana which was reported to be $91 \%$ (26).

Over half percentage of the prescribed drugs were adequately labeled with written information on; medication dose,frequency of adminstration and, in some cases, how to take the medicine. Even though only half were adequately labeled, paitent knowledge seems satisfactory. Despite the recommendation of WHO that a dispenser should have an adequate period with patients to provide basic drug orientation (21), the average dispensing time obtained in this study (53 seconds) seems to 
be short. Studies conducted in Ethiopia and elsewhere have also indicated that there is a problem in the time that pharmacists spend with patients $(6,27)$. Though patient overload might be one possible reason in the present setting, it is hardly possible for the patients or the caregiver to understand the instructions given by the dispenser and repeat back at least dose and frequency information in a minute.

In this study, absence of legal frame-work to monitor and evaluate patient counseling practice, high patient load and lack of time, lack of updated drug information, patient factors (either lack of patience to listen the counseling provided or poor attitude to pharmacy services) and, lack of space were barriers to patient counseling as the respondents reply. Similarly, in a study conducted in Nepal, lack of time, lack of knowledge, and lack of patient willingness were the barriers for patient counseling by dispensers (28). A study in North West Ethiopia has also reported high patient load, non-legalization of counseling and, patient factors as barriers to patient counseling (29). High percentage of absence of frame-work to monitor and evaluate patient counseling practice indicates that a guide should be established for the dispensers to properly perform the counseling activity. The percentage of lack of knowledge in this study showed that pharmacy professionals are in need of getting professional education.

In the present study, being a female has shown a twofold likelihood of being satisfied from pharmacist counseling services (OR: 2.33; 95\% CI: 1.31-4.13). This could be related to high tendency of females to seek medication counseling services and positive attitude to pharmacy services as scored from patient factors in the same study and females' curious attention to their medication as reported elsewhere (30). On the other hand, increase in level of education were found to have a negative association with pharmacist counseling service (OR: $0.20 ; \quad 95 \% \mathrm{CI}$ : 0.04-0.92; OR: $0.16 ; 95 \%$ CI: $0.03-0.75$ for grades $9-12$ and University or College respectively). The result might be attributed to unmet expectations in the counseling service as increase in level of education is a possible predictor of better knowledge on medication doses, side effects, administration and quality of counseling services
(31).

This study might be subjected to recall bias as it was performed, only, from the patients' perspective. Likely, the fact that medication counseling practices were observed in front of the pharmacy professionals might incur bias to the results obtained. The study also employed only a quantitative data collection approach and was limited, solely, to Tikur-Anbessa Specialized Hospital in Addis Ababa which may restrict the generalizability of the results to all hospitals in the country.

In conclusion, drug information on dose, frequency of administration and duration of therapy were the most frequently delivered information to patients. Majority of the patients reported that they were satisfied by the services given. However, the mean dispensing time in this study was too short to allow for optimal information to be communicated on drugs as well as for answering questions raised by patients.

\section{ACKNOWLEDMENTS}

We would like to take this opportunity to express our profound gratitude and deep regard to the outpatients of Tikur-Anbessa Specialized Hospital for their sincere interest and verbal consent to participate in this study.

\section{REFERENCES}

1. FMACHA. Manual for Medicines Good Dispensing Practice. 2012.

2. WHO. Policy Perspective on Medicines: Promoting rational use of medicines; Core components, Geneva, 2002.

3. Siti Maisharah S.G, Sabariah Noor H, Nur Hafzan M.H. Gillani SW. Outpatient Pharmacy Department (OPD) And Counseling Clerkship Among Final Year Pharmacy Undergraduates: Students' 
Expectation and Satisfaction. Int $J$ Pharm

Pharm Sci. 2012; Vol 4, Suppl 5, 484-489.

4. Kumud K. Farai C. and Suryawadi S. Role of dispensers in promoting rational drug use, ensuring good dispensing practice, 1996; 11(3):1-21.

5. Directorate General of Pharmacy (DGoP), Federal Ministry of Health. Dispensing \& Counseling. Sudan Journal of Rational Use of Medicine.2014; (10), Pages:1-34. available at: http://apps.who.int/medicinedocs/documents/s 22243en/s22243en.pdf

6. Ayalew M. Medication Counseling Practice in Ethiopia: A Systematic Review. J Basic Clin Pharma. 2017;8:S001-S005.

7. Beardsley R. Oral patient counseling by pharmacists; proceeding of the national symposium on oral counseling by pharmacist about prescription medicines. 1997; 19-21.

8. Chaturvedi VP, Mathur AG, Anand AC. Rational drug use - As common as common sense?. Med $J$ Armed Forces India. 2012;68(3):206-8.

9. Richardson WC, Berwick D, Bisgard J, Bristow L, Buck C, Cassel C. Crossing the quality chasm: a new health system for the 21st century: Institute of Medicine. Washington: National Academy Press; 2001.

10. Melanic J. Pharmacists talking with patients. A Guide to patient counseling, 2nd edition. 2007; 68-72.

11. Schnipper JL, Kirwin JL, Cotugno MC, Wahlstrom SA, Brown BA, Tarvin E, et al. Role of pharmacist counseling in preventing adverse drug events after hospitalization. Arch Intern Med. 2006;166565- 57.1

12. Daliri S, Hugtenburg JG, Ter Riet G, Van Den Bemt BJF, Buurman BM, Scholte Op Reimer WJM, et al. The effect of a pharmacy-led transitional care program on medicationrelated problems post-discharge: A beforeAfter prospective study. PLoS One. 2019 Mar 12;14(3):e0213593.

doi:

10.1371/journal.pone.

13. Hanlon JT, Lindblad CI, Gray SL. Can clinical pharmacy services have a positive impact on drug-related problems and health outcomes in community-based older adults? Am J Geriatr Pharmacother. 2004; 2(1):3-13.

14. Alfadl AA, Alrasheedy AA, Alhassun MS. Evaluation of medication counseling practice at community pharmacies in Qassim region, Saudi Arabia. Saudi Pharmaceutical Journal. 2018; 26(2):258-262.

15. Teka NT, Baye AM. Counseling practice of community pharmacists for diabetes mellitus patients in Addis Ababa, Ethiopia. BMC Res Notes. 2018;11(1):700. Published 2018 Oct 4. doi:10.1186/s13104-018-3807-6.

16. Berhe DF, Taxis K, Haaijer-Ruskamp FM, Mol PGM. Healthcare professionals' level of medication knowledge in Africa: a systematic review. $\mathrm{Br} J$ Clin Pharmacol. 2018;84(12):2729-2746. doi:10.1111/bcp.13746.

17. Nigussie WD. Patient Counselling at Dispensing of Medicines in Health Care Facility Outpatient Pharmacies of Bahir Dar City, Northwest Ethiopia. Science Journal of Public Health. 2014; 2(2):126-134. doi: 10.11648/j.sjph.20140202.22.

18. Bruce Bayley K, Savitz LA, Maddalone T, Stoner SE, Hunt JS, Wells R. Evaluation of patient care interventions and recommendations by a transitional care pharmacist. Ther Clin Risk Manag. 2007;3(4):695-703.

19. Belachew SA, Yimenu DK, Gebresillassie BM. Pharmacy Professionals' Dispensing Practice, Knowledge, and Attitude towards Emergency Contraceptives in Gondar Town, Northwestern Ethiopia: A Cross-Sectional Study. International Journal of Reproductive 
Medicine. Vol.2017, Article ID 8754126, 9 pages. doi.org/10.1155/2017/8754126.

20. Belay YB, Kassa TT, Welie AG, Alemayehu MS. Assessment of counseling practice in medicine retail outlets in Mekelle City, Northern Ethiopia. Risk Manag Healthc Policy. 2017;10:137-146. doi: 10.2147/RMHP.S138300. eCollection 2017.

21. WHO. How to investigate drug use in health facilities: Selected drug use indicators. WHO/DAP/93.1:1-97 Geneva; 1993.

22. Hanna K., N. D. Assessment of patient knowledge about their long term therapy. European journal of nursing. 2004 .

23. Alefe $\mathrm{N}$ et al. Assessment of Knowledge, Attitude and Practice of Patient Medication Counseling Among Drug Dispensers in Jimma Town, Oromia Region, South West Ethiopia. Indo American Journal of Pharm Research. 2014:4(06):2817-2826.

24. Sina A, Norah OA. Counselling practices in community pharmacies in Riyadh, Saudi Arabia: a cross-sectional study. BMC Health Services Research. 2015;15:557-66.

25. Azhar H, Mohamed I. Medication counselling and dispensing practices at community pharmacies: a comparative cross-sectional study from Pakistan. Int $J$ Clin Pharm. 2011;33:859-67. 